\title{
Doubly-charged Higgs boson at a future electron-proton collider
}

\author{
P. S. Bhupal Dev, ${ }^{1, *}$ Sarif Khan, ${ }^{2,3,4, \dagger}$ Manimala Mitra, ${ }^{5,3, *}$ and Santosh Kumar Rai ${ }^{2,6,3,8}$ \\ ${ }^{1}$ Department of Physics and McDonnell Center for the Space Sciences, Washington University, \\ St. Louis, Missouri 63130, USA \\ ${ }^{2}$ Harish-Chandra Research Institute, Chhatnag Road, Jhusi, Allahabad 211019, India \\ ${ }^{3}$ Homi Bhabha National Institute, Training School Complex, Anushakti Nagar, Mumbai 400094, India \\ ${ }^{4}$ Institut für Theoretische Physik, Georg-August-Universität Göttingen, \\ Friedrich-Hund-Platz 1, Göttingen, D-37077 Germany \\ ${ }^{5}$ Institute of Physics, Sachivalaya Marg, Bhubaneswar 751005, India \\ ${ }^{6}$ Regional Centre for Accelerator-based Particle Physics, Harish-Chandra Research Institute, \\ Chhatnag Road, Jhusi, Allahabad 211019, India
}

(Received 13 March 2019; published 11 June 2019)

\begin{abstract}
We explore the discovery prospect of the doubly-charged component of an $S U(2)_{L}$-triplet scalar at the future $e^{-} p$ collider FCC-eh, proposed to operate with an electron beam energy of $60 \mathrm{GeV}$ and a proton beam energy of $50 \mathrm{TeV}$. We consider the associated production of the doubly-charged Higgs boson along with leptons and jet(s), and its subsequent prompt decay to same-sign lepton pair. This occurs for $\mathcal{O}(1)$ Yukawa coupling of the scalar triplet with charged leptons, which is expected for reasonably small vacuum expectation values of the neutral component of the triplet field that governs the neutrino mass generation in the type-II seesaw. We present our analysis for two different final states, $3 l+\geq 1 j$ and an inclusive $\geq 2 l+\geq 1 j$ channel. Considering its decay to electrons only, we find that the doubly-charged Higgs boson with a mass around a $\mathrm{TeV}$ could be observed at the $3 \sigma$ confidence level with $\mathcal{O}(200) \mathrm{fb}^{-1}$ of integrated luminosity, while masses up to $2 \mathrm{TeV}$ could be probed within a few years of data accumulation. The signal proposed here becomes essentially background free, if it is triggered in the $\mu \mu$ mode and a $5 \sigma$ discovery is achievable in this channel for a $\mathrm{TeV}$-scale doubly-charged Higgs boson with an integrated luminosity as low as $\mathcal{O}(50) \mathrm{fb}^{-1}$. We also highlight the sensitivity of FCC-eh to the Yukawa coupling responsible for the production of the doubly-charged Higgs boson as a function of its mass in both the $e e$ and $\mu \mu$ channels.
\end{abstract}

DOI: 10.1103/PhysRevD.99.115015

\section{INTRODUCTION}

The observations of flavor-changing neutrinos in neutrino oscillation experiments have conclusively established that neutrinos have nonzero masses and mixing. Specifically, the solar and atmospheric mass square differences are measured to be $\Delta m_{12}^{2} \sim 7.6 \times 10^{-5} \mathrm{eV}^{2}$, and $\left|\Delta m_{13}^{2}\right| \sim 2.5 \times 10^{-3} \mathrm{eV}^{2}$, and the mixing angles are $\theta_{12} \sim 33^{\circ}, \theta_{23} \sim 42^{\circ}$, and $\theta_{13} \sim 8^{\circ}$ [1]. These experimental observations can not be explained within the framework of the Standard Model (SM), and hence, some beyond the Standard Model (BSM) extension is required. So far, a number of BSM models have been proposed to explain

\footnotetext{
*bdev@wustl.edu

†sarifkhan@hri.res.in

*manimala@iopb.res.in

§skrai@hri.res.in
}

Published by the American Physical Society under the terms of the Creative Commons Attribution 4.0 International license. Further distribution of this work must maintain attribution to the author(s) and the published article's title, journal citation, and DOI. Funded by SCOAP ${ }^{3}$. small neutrino masses [2]. Among them, one of the most appealing frameworks for light neutrino mass generation is the so-called seesaw mechanism, where the lepton-numberviolating (LNV) dimension-5 Weinberg operator $L L H H / \Lambda$ [3,4] (where $L$ and $H$ are, respectively, the SM lepton and Higgs doublets, and $\Lambda$ is the LNV scale) generates Majorana masses of light neutrinos. There are three treelevel realizations of seesaw [5], namely, type-I [6-10], type-II [11-16], and type-III [17] seesaw, depending on whether we add $S U(2)_{L}$-singlet fermions or $S U(2)_{L}$-triplet scalars or $S U(2)_{L}$-triplet fermions to the SM particle content, respectively. One can also have hybrid combinations, such as type-I+type-II seesaw [12], which naturally occurs, for instance, in the left-right symmetric extension of the SM [18-20]. There also exist several other variations of the minimal seesaw, such as the inverse [21-23], linear [24], and extended [25-28] seesaw, as well various radiative neutrino mass generation mechanisms [29]. If the scale of the dimension-5 Weinberg operator for neutrino mass generation is within a few $\mathrm{TeV}$ range, the seesaw mechanism can be tested by looking for LNV signatures in current and future colliders [30-34]. 
In this paper, we focus on the LNV signatures of seesaw at future $e^{-} p$ colliders, such as LHeC [35] and FCC-eh [36]. Although their center-of-mass energy is down with respect to the $p p$ option by a factor of $\sqrt{E_{p} / E_{e}} \sim 10(30)$ for $\mathrm{LHeC}$ (FCC-eh), the $e^{-} p$ colliders are capable of exploring a broad range of BSM scenarios and characterizing BSM hints at $e e$ and $p p$ colliders [37]. There have been quite a few studies on the prospects of the $S U(2)_{L}$-singlet heavy neutrino production at future $e^{-} p$ colliders [38-48]. Here, we explore the prospects of the $S U(2)_{L}$-triplet scalar $(\Delta)$, and in particular, the production and decay of the doubly-charged component $\left(H^{ \pm \pm}\right)$of the triplet at a future $e^{-} p$ collider. For earlier studies of the doubly-charged Higgs production in $e^{+} p$ collision at HERA, see Refs. [49-51]. The triplet Higgs boson acquires an induced vacuum expectation value (VEV) $v_{\Delta}$ and generates light neutrino masses at tree-level via the type-II seesaw mechanism [12-16]. We consider $v_{\Delta}$ to be small, i.e., $v_{\Delta} \sim \mathcal{O}(0.1) \mathrm{eV}$, in the same ballpark as the upper limit on the sum of neutrino masses from cosmology [52]. For this choice of the triplet VEV, the Yukawa couplings of the triplet scalar $\Delta$ with a SM lepton-pair are large: $Y^{\Delta} \sim \mathcal{O}(1)$. This is where the production of a doubly-charged scalar in $e^{-} p$ collision has a major difference when compared with its production in $p p$ collision. At the LHC, the dominant production modes for the doublycharged Higgs boson are pair production via the $s$ channel $\gamma / Z$ and associated production with a singly-charged Higgs boson via the $s$-channel $W$ exchange [53-76]. The pair production through $\gamma \gamma$ fusion is also important for higher doubly-charged scalar masses [77], whereas the single production through $W W$ fusion [78-85] is suppressed by the smallness of the triplet VEV $v_{\Delta}$ and is not accessible to the LHC [85]. Due to the hadrophobic nature of the triplet scalar, one can never exploit the large Yukawa $Y^{\Delta}$ to enhance the production cross section of $\Delta$ fields at hadron colliders, although the size and structure of $Y^{\Delta}$ still play an important role in deciding their dominant decay modes. In contrast, at the $e^{-} p$ collider, the doubly-charged scalar can be singly produced in association with a lepton $\left(l\right.$ or $\left.\nu_{l}\right)$ and a jet (see Fig. 1), with the cross section directly proportional to the square of the Yukawa coupling to the electron flavor, $\left|Y_{e l}^{\Delta}\right|^{2}$ (with $l=e, \mu, \tau$ ). Therefore, as we show in this paper, the Yukawa coupling $Y^{\Delta}$ can be directly measured at a future $e^{-} p$ collider, as long as it is reasonably large $\sim \mathcal{O}(1)$ to give an observable signal-to-background ratio. This is largely complementary to the case of future lepton colliders, where the doubly-charged scalar can also be singly/pair produced via the Yukawa coupling $Y_{e l}^{\Delta}$ [86-94].

For large diagonal Yukawa couplings, the off diagonal entries of $Y^{\Delta}$ are restricted from LFV processes, such as $\mu \rightarrow 3 e, \mu \rightarrow e \gamma$, and $\mu-e$ conversion in nuclei [75,94-96]. To evade this conflict, we consider the scenario with a large diagonal $Y_{i i}^{\Delta}$ (with $i=e, \mu$ ) and small off diagonal $Y_{i j}^{\Delta}$
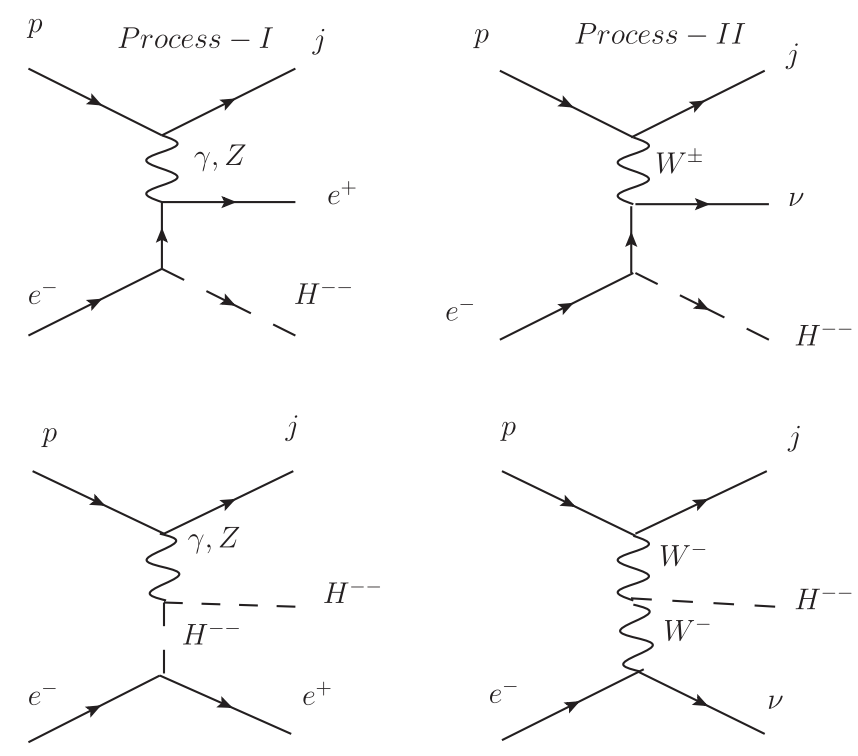

FIG. 1. Feynman diagrams for the single production of doublycharged Higgs boson at the $e^{-} p$ collider. An additional diagram exists for process $I$ with the $t$-channel photon exchange appearing before the doubly-charged Higgs boson is radiated, which is not shown here but included in our analysis.

(with $i \neq j$ ) that are in agreement with LFV measurements and are also promising for collider searches of $H^{ \pm \pm}$at an $e^{-} p$ collider. However, this choice can not reproduce the observed neutrino mixing angles, which require a highly nondiagonal PMNS mixing matrix. The conflict with the neutrino data can be evaded by considering variations in the pure type-II seesaw model. A simple possibility is to consider a hybrid seesaw model, that contains both the gauge singlet, right-handed (rh) neutrinos and the triplet Higgs boson. The light neutrino mass is generated by both singlet neutrino (type-I) and the triplet Higgs (type-II) contributions. The phenomenology of the singlet neutrinos at the $e^{-} p$ collider will be the same as discussed before $[41,44]$, and our main interest here lies in the doublycharged Higgs phenomenology.

The direct search constraints on the doubly-charged scalar from the LHC put a lower bound on $M_{H^{ \pm \pm}} \gtrsim$ $800 \mathrm{GeV}$ at $95 \%$ C.L. $[97,98]$. For the dominant $H^{ \pm \pm}$ production channels considered here, we find that the proposed LHeC with $\sqrt{s}=1.3 \mathrm{TeV}$ (or even the highenergy $\mathrm{LHeC}$ with $\sqrt{s}=1.8 \mathrm{TeV}$ ) cannot probe the doubly-charged Higgs boson beyond its current LHC limit. Therefore, for the collider analysis, we consider the future circular collider-based $e^{-} p$ scenario (FCC-eh) with $\sqrt{s}=3.46 \mathrm{TeV}$. We focus on two different signals: (i) $3 l+\geq 1 j$, and (ii) an inclusive search for $H^{--}$in the $\geq 2 l+\geq 1 j$ final state, with at least two negatively-charged leptons of same flavor in each case. We consider a number of SM backgrounds that can mimic our signal. By choosing suitable cuts on the kinematic variables, we reduce the background significantly and achieve an observable 
signal-to-background ratio. We show that doubly-charged Higgs masses up to $2 \mathrm{TeV}$ can be probed at the $3 \sigma$ confidence level with $500 \mathrm{fb}^{-1}$ integrated luminosity at FCC-eh.

The rest of the paper is organized as follows. In Sec. II, we review the basic relevant features of the triplet Higgs model. In Sec. III, we discuss the dominant production channels and the signals for doubly-charged scalar at $e^{-} p$ collider. Followed by this, we present a detailed event analysis using cut-based techniques for the signal and background at FCC-eh. Finally, we present our conclusions in Sec. IV.

\section{TRIPLET HIGGS MODEL}

We consider a hybrid type-I + type-II seesaw model, where the SM particle content is extended by an additional $S U(2)_{L}$-triplet scalar with hypercharge $Y=2$,

$$
\Delta=\left(\begin{array}{cc}
\frac{\delta^{+}}{\sqrt{2}} & \delta^{++} \\
\delta^{0} & -\frac{\delta^{+}}{\sqrt{2}}
\end{array}\right),
$$

and the SM gauge-singlet rh neutrinos $N_{R, i}$ (with $i=1,2,3$ being the family index). The complete particles spectrum is shown in Table I, along with the corresponding charges under the SM gauge group. Note that the presence of both type-I and type-II seesaw components can be naturally motivated as originating from a left-right symmetric gauge group, or even higher gauge groups like Pati-Salam or $S O(10)$ at a high scale.

The complete Lagrangian with the additional triplet scalar and rh neutrino is given by

$$
\mathcal{L}=\mathcal{L}_{\mathrm{SM}}+\mathcal{L}_{\text {kin }}^{N_{R}}+\operatorname{Tr}\left[\left(D_{\mu} \Delta\right)^{\dagger}\left(D^{\mu} \Delta\right)\right]-\mathcal{V}(\phi, \Delta)+\mathcal{L}_{\mathrm{Yuk}},
$$

where $\mathcal{L}_{\mathrm{SM}}$ is the complete Lagrangian for the SM particles and $\mathcal{L}_{\text {kin }}^{N_{R}}=i \bar{N}_{R} \gamma^{\mu} \partial_{\mu} N_{R}$ is the kinetic term of the righthanded neutrinos. The covariant derivative for the triplet Higgs boson takes the following form:

$$
D_{\mu} \Delta=\partial_{\mu} \Delta+\frac{i g}{2}\left[\sigma^{i} W_{\mu}^{i}, \Delta\right]+i g^{\prime} B_{\mu} \Delta,
$$

where $W_{\mu}^{i}, B_{\mu}$ are the $S U(2)_{L}$ and $U(1)_{Y}$ gauge fields, with the corresponding gauge couplings $g, g^{\prime}$, respectively, and $\sigma^{i}$ s (with $i=1,2,3$ ) are the Pauli matrices.

The Yukawa term in Eq. (2) takes the following form:

$$
\begin{aligned}
-\mathcal{L}_{\text {Yuk }}= & Y_{i j}^{\Delta} L_{i}^{T} C^{-1} \sigma_{2} \Delta L_{j}+Y_{i j}^{D} \bar{L}_{i} \tilde{\phi} N_{R, j} \\
& +\frac{1}{2} M_{R, i j} N_{R, i}^{T} C^{-1} N_{R, j}+\text { H.c. }
\end{aligned}
$$

where $C$ is the Dirac charge conjugation matrix with respect to the Lorentz group, $\tilde{\phi} \equiv i \sigma_{2} \phi^{*}$ and $Y^{D}$ is the Dirac Yukawa coupling for neutrinos. The first and third term in Eq. (4) violate lepton number by two units and give rise to the light neutrino mass at tree level via type-II and type-I seesaw mechanisms, respectively, after electroweak symmetry breaking. The neutral component of the Higgs doublet acquires a spontaneous VEV, while the neutral component of the Higgs triplet acquires an induced VEV,

$$
\langle\phi\rangle=\left(\begin{array}{c}
0 \\
\frac{v}{\sqrt{2}}
\end{array}\right), \quad\langle\Delta\rangle=\left(\begin{array}{cc}
0 & 0 \\
\frac{v_{\Delta}}{\sqrt{2}} & 0
\end{array}\right) .
$$

The Majorana neutrino mass matrix for the light neutrinos have the following form:

$$
M_{\nu}=M_{L}-M_{D}^{T} M_{R}^{-1} M_{D}
$$

where $M_{L}=\frac{v_{\Delta}}{\sqrt{2}} Y^{\Delta}$ and $M_{D}=\frac{v}{\sqrt{2}} Y^{D}$. In the limit $Y^{D} \rightarrow 0$, the light neutrino mass matrix becomes type-II dominant: $M_{\nu} \simeq \frac{v_{\Delta}}{\sqrt{2}} Y^{\Delta}$. Since the triplet VEV $v_{\Delta}$ governs the light neutrino mass in type-II seesaw, it is expected to be much smaller than the electroweak VEV $v$, i.e., $v_{\Delta} \ll v$. In fact, the electroweak precision data, and in particular, the $\rho$ parameter constraint requires that $v_{\Delta} \lesssim 2 \mathrm{GeV}$ [75]. For the collider study of doubly-charged Higgs production and decay (see Sec. III), we consider low VEV of the triplet Higgs boson, $v_{\Delta} \sim 0.1 \mathrm{eV}$, that generates large Yukawa $Y^{\Delta} \sim \mathcal{O}(1)$. In particular, we consider large diagonal

\begin{tabular}{|c|c|c|c|c|c|c|c|c|}
\hline \multirow[b]{2}{*}{ Gauge group } & \multicolumn{3}{|c|}{ Baryon fields } & \multicolumn{3}{|c|}{ Lepton fields } & \multicolumn{2}{|c|}{ Scalar fields } \\
\hline & $Q_{L, i}=\left(u_{L, i}, d_{L, i}\right)^{T}$ & $u_{R, i}$ & $d_{R, i}$ & $L_{i}=\left(\nu_{L, i}, e_{L, i}\right)^{T}$ & $e_{R, i}$ & $N_{R, i}$ & $\phi$ & $\Delta$ \\
\hline$S U(3)_{c}$ & 3 & 3 & 3 & 1 & 1 & 1 & 1 & 1 \\
\hline$S U(2)_{L}$ & 2 & 1 & 1 & 2 & 1 & 1 & 2 & 3 \\
\hline$U(1)_{Y}$ & $1 / 6$ & $2 / 3$ & $-1 / 3$ & $-1 / 2$ & -1 & 0 & $1 / 2$ & 1 \\
\hline
\end{tabular}
elements of $Y_{i i}^{\Delta}$ (with $i=e, \mu$ ), and small off diagonal Yukawa $Y_{i j}^{\Delta}$ (with $i \neq j$ ) in order to avoid the bounds from LFV processes mediated by the doubly-charged scalars $[75,95,96,99,100]$. Therefore, we need the type-I seesaw

TABLE I. Particle content and their corresponding charges under the SM gauge group. The $U(1)_{Y}$ hypercharges in the last row correspond to $Y / 2$. Here, $i=1,2,3$ stands for the family index. 
contribution in Eq. (6) to reproduce the observed pattern of the Pontecorvo-Maki-Nakagawa-Sakata (PMNS) mixing matrix in the light neutrino sector. Apart from fitting the neutrino mass, the Dirac Yukawa couplings $Y^{D}$ play no other role in our subsequent discussion.

The scalar potential in Eq. (2) has the following form:

$$
\begin{aligned}
\mathcal{V}(\phi, \Delta)= & -\mu_{\phi}^{2}\left(\phi^{\dagger} \phi\right)+\frac{\lambda}{4}\left(\phi^{\dagger} \phi\right)^{2}-\mu_{\Delta}^{2} \operatorname{Tr}\left[\Delta^{\dagger} \Delta\right] \\
& +\left[\mu \phi^{T} i \sigma_{2} \Delta^{\dagger} \phi+\text { H.c. }\right] \\
& +\lambda_{1}\left(\phi^{\dagger} \phi\right) \operatorname{Tr}\left[\Delta^{\dagger} \Delta\right]+\lambda_{2}\left(\operatorname{Tr}\left[\Delta^{\dagger} \Delta\right]\right)^{2} \\
& +\lambda_{3} \operatorname{Tr}\left[\left(\Delta^{\dagger} \Delta\right)^{2}\right]+\lambda_{4}\left(\phi^{\dagger} \Delta \Delta^{\dagger} \phi\right) .
\end{aligned}
$$

In the above potential, one can introduce another term $\lambda_{5}\left(\phi^{\dagger} \Delta^{\dagger} \Delta \phi\right)$, but this quartic coupling $\lambda_{5}$ can be easily absorbed by redefining the couplings $\lambda_{1}$ and $\lambda_{4}$, by virtue of the relation $\phi^{\dagger} \Delta \Delta^{\dagger} \phi+\phi^{\dagger} \Delta^{\dagger} \Delta \phi=\phi^{\dagger} \phi \operatorname{Tr}\left[\Delta^{\dagger} \Delta\right]$, which is valid because $\Delta$ is a traceless $2 \times 2$ matrix [cf. Eq. (1)].

We expand the doublet and triplet scalar fields around their VEVs to obtain ten real-valued field components,

$$
\phi=\left(\begin{array}{c}
\phi^{+} \\
\frac{v+h_{0}+i \xi_{1}}{\sqrt{2}}
\end{array}\right), \quad \Delta=\left(\begin{array}{cc}
\frac{\delta^{+}}{\sqrt{2}} & \delta^{++} \\
\frac{v_{\Delta}+\delta_{0}+i \xi_{2}}{\sqrt{2}} & -\frac{\delta^{+}}{\sqrt{2}}
\end{array}\right) .
$$

We consider $\mu_{\phi}^{2}>0$ and $\mu_{\Delta}^{2}>0$ in Eq. (7). We also assume the parameter $\mu$ in the cubic term of Eq. (7) to be real. Upon minimization of the scalar potential with respect to the VEVs, we get the following relations:

$\mu_{\Delta}^{2}=\frac{2 \mu v^{2}-\sqrt{2}\left(\lambda_{1}+\lambda_{2}\right) v^{2} v_{\Delta}-2 \sqrt{2}\left(\lambda_{2}+\lambda_{3}\right) v_{\Delta}^{3}}{2 \sqrt{2} v_{\Delta}}$,

$\mu_{\phi}^{2}=\frac{\lambda v^{2}}{4}-\sqrt{2} \mu v_{\Delta}+\frac{\lambda_{1}+\lambda_{4}}{2} v_{\Delta}^{2}$,

and the mass terms for the scalar fields. The mass term of the doubly-charged Higgs boson has the following form:

$$
M_{H^{ \pm \pm}}^{2}=\frac{\sqrt{2} \mu v^{2}-\lambda_{4} v^{2} v_{\Delta}-2 \lambda_{3} v_{\Delta}^{3}}{2 v_{\Delta}},
$$

where we have defined the mass basis of the doublycharged Higgs boson as $\delta^{ \pm \pm}=H^{ \pm \pm}$.

The mass matrix of the singly-charged Higgs boson in the basis $\left(\phi^{ \pm}, \delta^{ \pm}\right)$takes the following form:

$$
M_{ \pm}^{2}=\left(\sqrt{2} \mu-\frac{\lambda_{4} v_{\Delta}}{2}\right)\left(\begin{array}{cc}
v_{\Delta} & -\frac{v}{\sqrt{2}} \\
-\frac{v}{\sqrt{2}} & \frac{v^{2}}{2 v_{\Delta}}
\end{array}\right) .
$$

We define the mass basis for the singly-charged scalars as $\left(G^{ \pm}, H^{ \pm}\right)$, which are related to the Lagrangian fields by an orthogonal matrix parametrized by the mixing angle $\beta^{\prime}$ with $\tan \beta^{\prime}=\frac{\sqrt{2} v_{\Delta}}{v}$ in the following way:

$$
\left(\begin{array}{c}
G^{ \pm} \\
H^{ \pm}
\end{array}\right)=\left(\begin{array}{cc}
\cos \beta^{\prime} & \sin \beta^{\prime} \\
-\sin \beta^{\prime} & \cos \beta^{\prime}
\end{array}\right)\left(\begin{array}{c}
\phi^{ \pm} \\
\delta^{ \pm}
\end{array}\right)
$$

After diagonalization, we get the following eigenvalue for the physical singly-charged scalar:

$$
M_{H^{ \pm}}^{2}=\frac{\left(v^{2}+2 v_{\Delta}^{2}\right)\left(2 \sqrt{2} \mu-\lambda_{4} v_{\Delta}\right)}{4 v_{\Delta}},
$$

whereas $M_{G^{ \pm}}^{2}=0$, corresponding to the Goldstone bosons $G^{ \pm}$responsible for the mass of the singly-charged gauge bosons $W^{ \pm}$. In the limit $v_{\Delta} \ll v$, using Eq. (9), we can relate the doubly- and singly-charged scalar masses in Eqs. (11) and (14) as follows:

$$
M_{H^{ \pm \pm}}^{2} \simeq M_{H^{ \pm}}^{2}-\frac{1}{2} \lambda_{4} v^{2}
$$

We assume the quartic coupling $\lambda_{4} \ll 1$, so that the cascade decay of $H^{ \pm \pm} \rightarrow H^{ \pm} W^{ \pm(*)}$ is kinematically suppressed, as compared to the dilepton decay mode $H^{ \pm \pm} \rightarrow l^{ \pm} l^{ \pm}$. The diboson channel $H^{ \pm \pm} \rightarrow W^{ \pm} W^{ \pm}$is always suppressed, as long as $v_{\Delta} \lesssim 0.1 \mathrm{MeV}$ [59].

Among the four neutral scalars, two of them are $C P$ even and the remaining two are $C P$ odd, as can be seen from Eq. (8). Assuming $C P$ conservation, we can write the $C P$-even and $C P$-odd mass matrices as follows:

$$
\begin{aligned}
M_{\mathrm{even}}^{2} & =\left(\begin{array}{cc}
\frac{\lambda v^{2}}{2} & -\sqrt{2} \mu v+\left(\lambda_{1}+\lambda_{4}\right) v_{\Delta} v \\
-\sqrt{2} \mu v+\left(\lambda_{1}+\lambda_{4}\right) v_{\Delta} v & \frac{\sqrt{2} \mu v^{2}+4\left(\lambda_{2}+\lambda_{3}\right) v_{\Delta}^{3}}{2 v_{\Delta}}
\end{array}\right) \\
& \equiv\left(\begin{array}{ll}
A & B \\
B & C
\end{array}\right),
\end{aligned}
$$

$M_{\mathrm{odd}}^{2}=\sqrt{2} \mu\left(\begin{array}{cc}2 v_{\Delta} & -v \\ -v & \frac{v^{2}}{2 v_{\Delta}}\end{array}\right)$.

We define the mass eigenbasis both for $C P$-even and $C P$ odd scalars, which are related to the Lagrangian fields in the following manner:

$$
\begin{aligned}
& \left(\begin{array}{c}
h \\
H^{0}
\end{array}\right)=\left(\begin{array}{cc}
\cos \alpha & \sin \alpha \\
-\sin \alpha & \cos \alpha
\end{array}\right)\left(\begin{array}{c}
h_{0} \\
\delta_{0}
\end{array}\right) \text { and } \\
& \left(\begin{array}{c}
G^{0} \\
A^{0}
\end{array}\right)=\left(\begin{array}{cc}
\cos \beta & \sin \beta \\
-\sin \beta & \cos \beta
\end{array}\right)\left(\begin{array}{l}
\xi_{1} \\
\xi_{2}
\end{array}\right) .
\end{aligned}
$$

Here, $h$ represents the SM-like Higgs boson and $G^{0}$ represents the Goldstone boson associated with the SM $Z$ boson. The mixing angles related to the neutral fields depend on the VEVs and the quartic couplings in the following manner: 
$\tan 2 \alpha=\frac{2 B}{A-C}, \quad$ and $\quad \tan \beta=\frac{2 v_{\Delta}}{v}=\sqrt{2} \tan \beta^{\prime}$.

The eigenvalues of the mass matrices (16) and (17) are given by

$$
\begin{aligned}
M_{h}^{2} & =\frac{1}{2}\left[A+C-\sqrt{(A-C)^{2}+4 B^{2}}\right], \\
M_{H^{0}}^{2} & =\frac{1}{2}\left[A+C+\sqrt{(A-C)^{2}+4 B^{2}}\right], \\
M_{A^{0}}^{2} & =\frac{\mu\left(v^{2}+4 v_{\Delta}^{2}\right)}{\sqrt{2} v_{\Delta}}, \\
M_{G^{0}}^{2} & =0 .
\end{aligned}
$$

We can easily write down the quartic couplings and the $\mu$ parameter appearing in Eq. (7) in terms of the abovementioned physical masses,

$$
\begin{aligned}
\lambda_{1}= & -\frac{2}{v^{2}+4 v_{\Delta}^{2}} M_{A^{0}}^{2}+\frac{4}{v^{2}+2 v_{\Delta}^{2}} M_{H^{ \pm}}^{2}+\frac{\sin 2 \alpha}{2 v v_{\Delta}}\left(M_{h}^{2}-M_{H^{0}}^{2}\right), \\
\lambda_{2}= & \frac{1}{v_{\Delta}^{2}}\left[\frac{\sin ^{2} \alpha M_{h}^{2}+\cos ^{2} \alpha M_{H^{0}}^{2}}{2}+\frac{v^{2} M_{A^{0}}^{2}}{2\left(v^{2}+4 v_{\Delta}^{2}\right)}\right. \\
& \left.-\frac{2 v^{2} M_{H^{ \pm}}^{2}}{v^{2}+2 v_{\Delta}^{2}}+M_{H^{ \pm \pm}}^{2}\right] \\
\lambda_{3}= & \frac{1}{v_{\Delta}^{2}}\left[\frac{-v^{2} M_{A^{0}}^{2}}{v^{2}+4 v_{\Delta}^{2}}+\frac{2 v^{2} M_{H^{ \pm}}^{2}}{v^{2}+2 v_{\Delta}^{2}}-M_{H^{ \pm \pm}}^{2}\right] \\
\lambda_{4}= & \frac{4 M_{A^{0}}^{2}}{v^{2}+4 v_{\Delta}^{2}}-\frac{4 M_{H^{ \pm}}}{v^{2}+2 v_{\Delta}^{2}}, \\
\lambda= & \frac{2}{v^{2}}\left[\cos ^{2} \alpha M_{h}^{2}+\sin ^{2} \alpha M_{H^{0}}^{2}\right] \\
\mu & \frac{\sqrt{2} v_{\Delta} M_{A^{0}}^{2}}{v^{2}+4 v_{\Delta}^{2}} .
\end{aligned}
$$

There are miscellaneous constraints on the quartic couplings from electroweak precision measurements, absence of tachyonic modes, boundedness of the potential, unitarity, vacuum stability, and naturalness considerations [70,96,101-108]; all of which have been taken into account in choosing the benchmark points in our subsequent collider analysis.

\section{SIGNATURES AT $e^{-} p$ COLLIDER}

In this section, we analyze the discovery prospects of a doubly-charged Higgs boson in the future $e^{-} p$ collider. We consider the FCC-eh design with beam energies $60 \mathrm{GeV}$ (for electron) and $50 \mathrm{TeV}$ (for proton), so the center-ofmass energy is $\sqrt{s} \simeq \sqrt{4 E_{e} E_{p}}=3.46 \mathrm{TeV}$, and a TeVscale doubly-charged Higgs boson can be produced on shell, through associated production, along with a charged lepton or neutrino and an energetic jet. We consider the following two production modes (see Fig. 1):

(i) Process I: $e^{-} p \rightarrow e^{+} j H^{--}, H^{--} \rightarrow l^{-} l^{-}$,

(ii) Process II: $e^{-} p \rightarrow j \nu H^{--}, H^{--} \rightarrow l^{-} l^{-}$,

where $l=e, \mu$. The diagrams in Fig. 1 clearly show the dominant production mode, depending on the choice of the triplet VEV. While the first three diagrams would be relevant for low values of the triplet VEV as it would lead to a larger Yukawa $Y_{e e}^{\Delta}$ coupling, they become less relevant when the VEV is chosen larger; in which case, the vectorboson-fusion (VBF) diagram becomes dominant. ${ }^{1}$ In process $I$, the contribution of $Z$ exchange is much smaller, as compared to the photon exchange. As for the photonmediated processes, depending on the photon virtuality, the proton may remain intact (elastic process) or dissociates (inelastic process). As discussed below, a selection cut of $40 \mathrm{GeV}$ on the transverse momentum of the final state jet is imposed in our analysis. This implies large photon virtuality (compared to the mass of the hadronic final state), and hence, the inelastic process, where the interaction involves a quark inside the proton, gives the dominant contribution.

It is worthwhile to note that the production of a doublycharged scalar at FCC-eh has a major difference when compared with its production at the LHC. At the LHC, one can never exploit the large Yukawa $Y_{e e}^{\Delta}$, although it still plays a very effective role in deciding the dominant decay modes for the doubly-charged Higgs boson. Notably, large $Y^{\Delta}$ at the LHC leads to the heavier-flavor charged lepton (tau) to be pair-produced more effectively from the $H^{ \pm \pm}$ decay when the neutrino masses exhibit "normal hierarchy," whereas for "inverted hierarchy," the electron and muon flavors will be pair produced more copiously than the tau flavor. On the other hand, for $Y^{\Delta} \lesssim 10^{-7}$, either the decay of $H^{ \pm \pm} \rightarrow l^{ \pm} l^{ \pm}$is displaced [75,109] or the diboson decay modes, such as $H^{ \pm \pm} \rightarrow W^{ \pm} W^{ \pm}$, take over the dilepton mode [80], depending on the $H^{ \pm \pm}$mass. So $Y_{e e}^{\Delta}$ still has a secondary role in the phenomenology of doubly-charged Higgs boson at hadron colliders, but cannot be directly probed due to its hadrophobic nature. In this work, we explore this region of parameter space where the triplet VEV is small, $v_{\Delta} \sim \mathcal{O}(0.1) \mathrm{eV}$, and $Y_{e e}^{\Delta}$ can be directly probed, as it plays the all important role in the doubly-charged scalar phenomenology at the FCC-eh.

It is also worth noting that we always produce the negatively-charged state at $e^{-} p$ colliders when produced singly. The decay of $\mathrm{H}^{--}$into two same-sign dileptons leads to the following signals, which we explore in detail:

${ }^{1}$ Thus, in the small VEV limit, our analysis equally applies to both $S U(2)_{L}$ and $S U(2)_{R}$ scalar triplets, such as in the left-right symmetric model. A polarized electron beam could, in principle, distinguish between the two contributions, by analyzing the angular distributions of the final-state leptons, for instance. We have only considered the unpolarized beam in this analysis, and the effect of polarization is left for future study. 
(i) Signal I: $3 l+\geq 1 j$ (with at least two negativelycharged leptons of same flavor),

(ii) Signal II: $\geq 2 l+\geq 1 j$ (where at least two negatively-charged leptons are of same flavor).

Note that signal I with a trilepton final state comes from the subprocesses listed as process I in Fig. 1 while signal II, which has at least two charged leptons can get contributions from all the subprocesses in Fig. 1, i.e., process $I$ and process II. Signal II also has substantial Missing Energy Transverse (MET) due to the presence of neutrinos in the final state as shown in process II. Although a trigger upon the MET could reduce the contributions from subprocesses in process I, which will have very little MET, it could prove useful in SM background suppression.

The production cross sections for $e^{-} p \rightarrow e^{+} j H^{--}$and $e^{-} p \rightarrow j \nu H^{--}$depend on the Yukawa coupling strength $Y_{e e}^{\Delta}$ quadratically. So we must choose large diagonal entries for $Y^{\Delta}$, as mentioned before. The off diagonal Yukawa couplings are then required to be quite small, so as to satisfy the LFV constraints from $\mu \rightarrow 3 e, \mu \rightarrow e \gamma$, etc. The current bounds on the branching ratio of $\mu \rightarrow e \gamma$ and $\mu \rightarrow$ eee are $4.2 \times 10^{-13}$ [110] and $10^{-12}$ [111], respectively. For our model, if the doubly-charged Higgs mass is around $1 \mathrm{TeV}$, then the corresponding bounds on the elements of $Y_{\alpha}^{\Delta}$ matrix are given by $[75,95,96]$

$$
\begin{aligned}
Y_{\mu e}^{\Delta} Y_{e e}^{\Delta} & <2.38 \times 10^{-5} \quad \text { and } \\
Y_{e e}^{\Delta} Y_{e \mu}^{\Delta}+Y_{e \mu}^{\Delta} Y_{\mu \mu}^{\Delta}+Y_{e \tau}^{\Delta} Y_{\mu \tau}^{\Delta} & <2.42 \times 10^{-4}
\end{aligned}
$$

Note that the diagonal elements $Y_{i i}^{\Delta}$ cannot be arbitrarily large either. Specifically, a large $Y_{e e}^{\Delta}$ would significantly contribute to the Bhabha scattering $e^{+} e^{-} \rightarrow e^{+} e^{-}$via the $t$ channel $H^{ \pm \pm}$, altering both the total cross section and the differential distributions [112-114]. Using the LEP data on the $e^{+} e^{-} \rightarrow e^{+} e^{-}$cross section measurements [113,115], we obtain a 90\% C.L. upper limit [93],

$$
\frac{\left|Y_{e e}^{\Delta}\right|^{2}}{M_{H^{ \pm \pm}}^{2}}<1.2 \times 10^{-7}
$$

Thus, for a TeV-scale $H^{ \pm \pm}$, we must have $\left|Y_{e e}^{\Delta}\right|<0.35$. $^{2}$ Similarly, both $Y_{e e}^{\Delta}$ and $Y_{\mu \mu}^{\Delta}$ contribute to the muoniumantimuonium oscillation, i.e., the LFV conversion of the bound states $\left(\mu^{+} e^{-}\right) \leftrightarrow\left(\mu^{-} e^{+}\right)$, whose oscillation probability has been constrained by the MACS experiment [116], and leads to the $90 \%$ C.L. upper limit [93],

$$
\frac{\left|\left(Y^{\Delta}\right)_{e e}^{\dagger} Y_{\mu \mu}^{\Delta}\right|}{M_{H^{ \pm \pm}}^{2}}<1.2 \times 10^{-7}
$$

\footnotetext{
${ }^{2}$ Note that the neutrinoless double beta decay limits are not relevant here, because the amplitude of the $H^{--}$-mediated process depends on the $H^{--} W^{+} W^{+}$coupling, which is suppressed by the small triplet VEV $v_{\Delta}$.
}

There exist other constraints involving the diagonal elements from $\mu \rightarrow e \gamma$ and electron/muon anomalous magnetic moment [93], which are however weaker than the two constraints mentioned above.

By choosing the diagonal elements $Y_{e e, \mu \mu}^{\Delta} \in[0.1,0.3]$ to be consistent with the bounds in (23) and (24), and assuming $Y_{\tau \tau}^{\Delta}$ to be small (since it does not play any role in our subsequent collider analysis), we show the allowed values of the off diagonal Yukawa coupling parameters $Y_{e \mu, e \tau, \mu \tau}^{\Delta}$ as a scatter plot in Fig. 2 which satisfy the LFV constraints shown in (22). Here, we have chosen $M_{H^{ \pm \pm}}=$ $1 \mathrm{TeV}$ as a benchmark value. The vertical and horizontal shaded regions are excluded from the experimental constraints on the tau-LFV decay modes $\tau \rightarrow 3 e$ and $\tau \rightarrow 3 \mu$, respectively [117], for fixed $Y_{e e, \mu \mu}^{\Delta}=0.3$. It is clear that the off diagonal Yukawa couplings are constrained to be small for our choice of large diagonal Yukawa couplings.

To simulate the signal events, we implement the model described in Sec. II in Feynrules [118] to create the UFO model files. The package Madgraph (v2.4.3) [119] is used to generate parton-level events using CTEQ6L1 [120] parton distribution functions for the colliding proton beam. The showering and subsequent hadronization has been done with Pythia (v6.4) [121]. Finally, we pass the generated events through Delphes (v3.4.1) [122] for detector simulation.

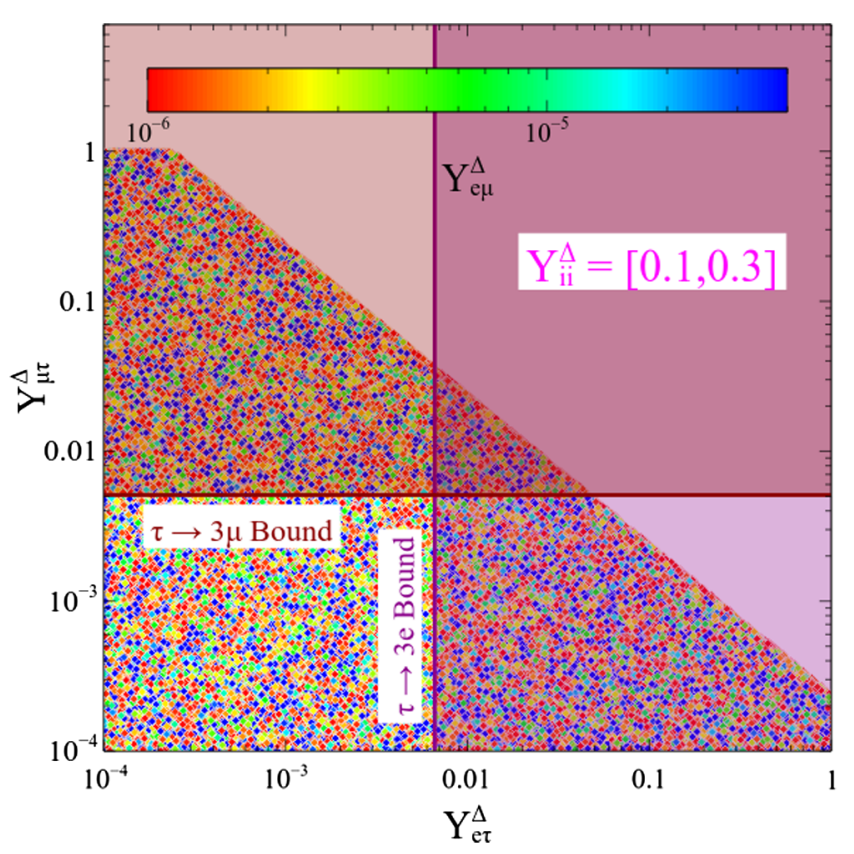

FIG. 2. Allowed off diagonal Yukawa couplings $Y_{\mu \tau}^{\Delta}$ and $Y_{e \tau}^{\Delta}$ as a function of $Y_{e \mu}^{\Delta}$ (shown by the color code). The diagonal couplings $Y_{e e, \mu \mu}^{\Delta}$ are randomly varied between the range of 0.1 and 0.3 , for a fixed $M_{H^{ \pm \pm}}=1 \mathrm{TeV}$. The vertical and horizontal shaded regions are excluded from the experimental constraints on the tau-LFV decay modes $\tau \rightarrow 3 e$ and $\tau \rightarrow 3 \mu$, respectively, for fixed $Y_{e e, \mu \mu}^{\Delta}=0.3$. 


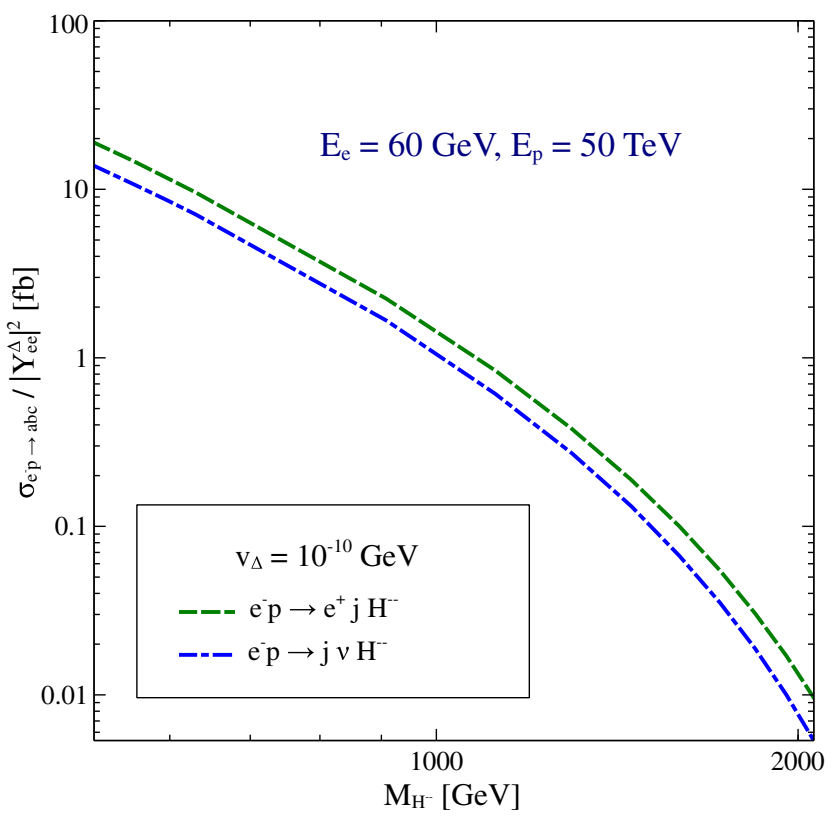

FIG. 3. Parton-level production cross sections (normalized to $\left.\left|Y_{e e}^{\Delta}\right|^{2}=1\right)$ of the doubly-charged Higgs boson for process I (green, dashed) and process II (blue, dot dashed) shown in Fig. 1 at $e^{-} p$ collider with beam energies of $60 \mathrm{GeV}$ (for electron) and $50 \mathrm{TeV}$ (for proton). We have chosen the triplet VEV as $v_{\Delta}=0.1 \mathrm{eV}$.

To reconstruct jets in the final state, we have used the anti- $k_{t}$ jet clustering algorithm [123] with a radius parameter $R=0.4$ in Fastjet [124] for jet formation.

We show the variation of the single production cross section of the doubly-charged Higgs boson as a function of its mass in Fig. 3 for both process I and process II. Note that process $I$ with a larger multiplicity of subprocesses as well as the enhanced coupling of the photon to the two units of charge of the doubly-charged scalar is significantly larger than the process II with $W$ boson exchange, where the second (VBF) subprocess hardly contributes to the production cross section due to the very small VEV. The mass range of interest for the doubly-charged Higgs boson for our analysis is dictated by the limits from $\sqrt{s}=13 \mathrm{TeV}$ LHC which is about $800 \mathrm{GeV}[97,98]$. As shown in Fig. 3, for $M_{H^{ \pm \pm}}$in between $500 \mathrm{GeV}$ and $1 \mathrm{TeV}$, the cross section varies between $20 \mathrm{fb}$ and $2 \mathrm{fb}$. For larger mass values, the cross section falls rapidly, as the mass approaches the center-of-mass energy of $\sqrt{s}=3.46 \mathrm{TeV}$ for the FCC-eh.

In Table II, we give the choice of our benchmark points which give us three different mass values for the doublycharged Higgs boson. A closer look at Eq. (11) shows that for a given choice of triplet VEV and $\lambda_{4}$, the value of $\mu$ would dictate the mass values. For simplicity, we just vary $\mu$ to generate different values for the doubly-charged scalar mass, while making sure that the quartic coupling values are consistent with all the theoretical constraints [70,96,101-108]. The first benchmark point we choose has
TABLE II. Representative benchmark points used in our analysis for studying the doubly-charged scalar production at the FCC-eh.

\begin{tabular}{|c|c|c|c|c|c|c|}
\hline $\begin{array}{l}\text { Benchmark } \\
\text { points }\end{array}$ & $\begin{array}{c}v_{\Delta} \\
{[\mathrm{GeV}]}\end{array}$ & $Y_{e e}^{\Delta}$ & $\mu[\mathrm{GeV}]$ & $\begin{array}{l}M_{H^{--}} \\
{[\mathrm{GeV}]}\end{array}$ & $\begin{array}{c}M_{H^{-}} \\
{[\mathrm{GeV}]}\end{array}$ & $\begin{array}{c}\Gamma_{H^{--}} \\
{[\mathrm{GeV}]}\end{array}$ \\
\hline BP1 & $10^{-10}$ & 0.31 & $2 \times 10^{-9}$ & 908.6 & 916.9 & 3.5 \\
\hline BP2 & $10^{-10}$ & 0.39 & $3 \times 10^{-9}$ & 1119.6 & 1126.2 & 6.8 \\
\hline BP3 & $10^{-10}$ & 0.45 & $4 \times 10^{-9}$ & 1296.7 & 1302.5 & 10.4 \\
\hline
\end{tabular}

a doubly-charged scalar mass close to the current LHC bound, which is kind of the most optimistic scenario for its discovery at FCC-eh. The $Y_{e e}^{\Delta}$ value chosen for each benchmark is the maximum value allowed by the LEP limit [cf. Eq. (23)] for the corresponding doubly-charged scalar mass. We also give the total decay width of the doubly-charged scalar in each case, which is simply given by $\Gamma_{H^{--}} \simeq \frac{\left|Y_{e e}^{\Delta}\right|^{2}}{8 \pi} M_{H^{--}}$, assuming that it dominantly decays to the ee final state only.

In what follows, we perform a detailed cut-based analysis for the signal and background for both signal I and signal II identified above. ${ }^{3}$

\section{A. Signal I $(3 l+\geq 1 j)$}

The trilepton signal containing at least a pair of samesign, same-flavor charged leptons can come from process I only. The SM background for the signal can have several sources, depending on their rate of production. We list the dominant and relevant SM processes below:

(1) The most dominant SM background for the above final state, at an $e^{-} p$ collider is the irreducible background given by $e^{-} p \rightarrow j e^{-} l^{+} l^{-}$.

(2) A subdominant contribution can also come from the reducible, on shell $Z$ boson production, which then decays to a pair of charged leptons via the process $e^{-} p \rightarrow e^{-} j Z$. Note that the requirement of a samesign, same-flavor pair of charged leptons in the final state ensures that only the $Z$ decay to $e^{-} e^{+}$ contributes.

(3) A relatively weaker background contribution could also come from an $\alpha_{\mathrm{em}}$-suppressed process $e^{-} p \rightarrow e^{-} j \gamma Z$, where the $Z$ boson again decays leptonically and specifically to $e^{-} e^{+}$only, while the photon is misidentified as a lepton. However, the photon misidentification rate is less than $10^{-4}$ for electron and $10^{-3}$ for muon [125], ${ }^{4}$ so this background will be negligible, as we will see below (in Table III).

\footnotetext{
${ }^{3}$ Since we consider the on shell production of the doublycharged scalar, and its width/mass is less than $1 \%$ (see Table II), the interference between signal and background is negligible.

${ }^{4}$ These numbers from Ref. [125] include both photons and jets misidentified as leptons. So the photon-only contribution will be even smaller.
} 
TABLE III. Cut-flow table of the cross section for the relevant SM background channels for the cuts A0-A3 as mentioned in the text at the FCC-eh collider with $3.46 \mathrm{TeV}$ center-of-mass energy. We assume $l=e$ or $\mu$.

\begin{tabular}{lccccc}
\hline \hline $\begin{array}{l}\text { SM backgrounds for } \\
\text { FCC-eh }\end{array}$ & & \multicolumn{3}{c}{$\begin{array}{c}\text { Effective cross section after } \\
\text { applying cuts }(\mathrm{fb})\end{array}$} \\
\cline { 1 - 5 } \cline { 4 - 7 } Channels & & $\mathbf{A 0}$ & $\mathbf{A 1}$ & $\mathbf{A 2}$ & $\mathbf{A 3}$ \\
\hline$e^{-} p \rightarrow e^{-} j \gamma Z\left(\rightarrow l^{+} l^{-}\right)$ & & 0.12 & 0.03 & 0.02 & $\ldots$ \\
$e^{-} p \rightarrow j e^{-} l^{+} l^{-}$ & & 53.13 & 12.57 & 3.63 & 0.08 \\
$e^{-} p \rightarrow e^{-} j j$ & & $104.08 \times 10^{3}$ & 0.2 & $\cdots$ & $\cdots$ \\
Total background & & & & & 0.08 \\
\hline \hline
\end{tabular}

(4) One should also account for the possibility of contributions to the SM background, where a jet may fake a charged lepton. Notwithstanding the fact that the fake-rate for such events would be significantly smaller, one begs the question whether a relatively sizable cross section for jet-enriched final state leaves an imprint on the signal under consideration. For the estimation of fake-rate induced background, we have considered the process $e^{-} p \rightarrow$ $e^{-} j j$ and assumed the rate for a jet faking a charged lepton as $0.1 \%$ [126].

To begin with, all the above channels for the background contribute substantially with basic acceptance cuts for the subprocess under consideration. However, the contributions to the actual signal topology starts showing the relative importance of the backgrounds that would eventually contribute to the final analysis. We begin the event generation by demanding:

A0. The final state leptons and jets satisfy a minimum requirement for the transverse momenta and their pseudorapidity, given by $p_{T}^{j}>40 \mathrm{GeV}, p_{T}^{l}>10 \mathrm{GeV}$, $\left|\eta^{j, l}\right| \leq 4.5$.

In Table III and Table IV, we present the cut-flow estimates for both the SM background and the signal cross section at the proposed FCC-eh machine. The A0 cuts are simply aimed at understanding the signal and background characteristics in a few kinematic variables which could be then utilized to device suitable cuts that help in

TABLE IV. Cut-flow table of cross section for signal for the cuts $\mathbf{A 0}-\mathbf{A 3}$ as mentioned in the text at the FCC-eh collider with $3.46 \mathrm{TeV}$ center-of-mass energy. Here, we assume $\operatorname{BR}\left(H^{ \pm \pm} \rightarrow e^{ \pm} e^{ \pm}\right)=100 \%$.

\begin{tabular}{|c|c|c|c|c|c|c|}
\hline \multicolumn{3}{|c|}{ Signal for FCC-eh } & \multicolumn{4}{|c|}{$\begin{array}{l}\text { Effective cross section after } \\
\text { applying cuts (fb) }\end{array}$} \\
\hline Process & Signal & $\mathrm{BP}$ & A0 & A1 & A2 & A3 \\
\hline I & $3 l+\geq 1 j$ & BP1 & 0.21 & 0.12 & 0.11 & 0.09 \\
\hline I & $3 l+\geq 1 j$ & BP2 & 0.13 & 0.07 & 0.07 & 0.06 \\
\hline I & $3 l+\geq 1 j$ & BP3 & 0.08 & 0.05 & 0.04 & 0.04 \\
\hline
\end{tabular}

improving the significance of the signal. Note that for the signal, the decay of the doubly-charged Higgs boson is an important variable. Since the off diagonal elements of $Y^{\Delta}$ are assumed to be small, only the lepton-flavorconserving modes $H^{ \pm \pm} \rightarrow l^{ \pm} l^{ \pm}$occur. As mentioned before, we shall neglect the $\tau$ decay mode and focus only on the $e^{-}$and $\mu^{-}$modes. We first consider the simple case where $H^{ \pm \pm}$decays to $e^{ \pm} e^{ \pm}$with $100 \%$ branching ratio; i.e., only the ee element of $Y^{\Delta}$ is large, and all other elements small. Later in Sec. III C, we will also consider a case where $H^{ \pm \pm}$decays to both $e^{ \pm} e^{ \pm}$and $\mu^{ \pm} \mu^{ \pm}$, each with $50 \%$ branching ratio; i.e., both $Y_{e e}^{\Delta}$ and $Y_{\mu \mu}^{\Delta}$ are of same size. This would help in generalizing the result when exploring the sensitivity for heavier mass values of the doubly-charged Higgs boson. To ensure that we only select electron or muon-flavored leptons, we impose a further requirement:

A1. The final state events must contain at least three charged leptons, among which we have $N\left(e^{+}\right)=1$, $N\left(\mu^{+}\right)=0$, and either a pair of $e^{-}$or $\mu^{-}$i.e., $N\left(e^{-}\right.$or $\left.\mu^{-}\right)=2$, while the number of jets in the final state must be greater than or equal to one, i.e., $N(j) \geq 1$.

This requirement is good enough to suppress most of the SM background, especially the jet-faking-lepton channel as seen from Table III. However, note that the doublycharged Higgs signal strength is totally dependent on the production cross section which depends on its mass. As LHC already puts strong limits, the mass range of our interest would entail a small production cross section even at the FCC-eh machine, evident in Fig. 3. Thus, we need to find methods of suppressing the background further to ensure sensitivity to heavier mass values of the doublycharged Higgs boson. The two very significant characteristics one expects are that the charged leptons coming from the decay of the heavy scalar would carry a significantly large transverse momentum and the invariant mass of the same-sign, same-flavor lepton pair would be concentrated in regions of very high mass, specific to the mass of the doubly-charged Higgs boson. In comparison, the SM background events should have leptons with relatively softer $p_{T}$, as well as a more continuum invariant mass distribution peaked at smaller values of $M_{l l}$.

In Fig. 4 , we show the transverse momentum $\left(p_{T}\right)$ distribution of the leading charged lepton, both for signal and background. Additionally, we also show the distribution of the invariant mass of the same-sign same-flavor leptons which shows expected differences between the signal and background events. We therefore impose the following cuts ( $\mathbf{A} 2$ and $\mathbf{A 3}$ ), which turn out to be very much useful in reducing the backgrounds events.

A2. We impose cuts on the transverse momentum of the leading and subleading charged lepton and also on the leading jet as: $p_{T}^{l_{i}}>50 \mathrm{GeV}(i=1,2)$ and $p_{T}^{j_{1}}>50 \mathrm{GeV}$. 

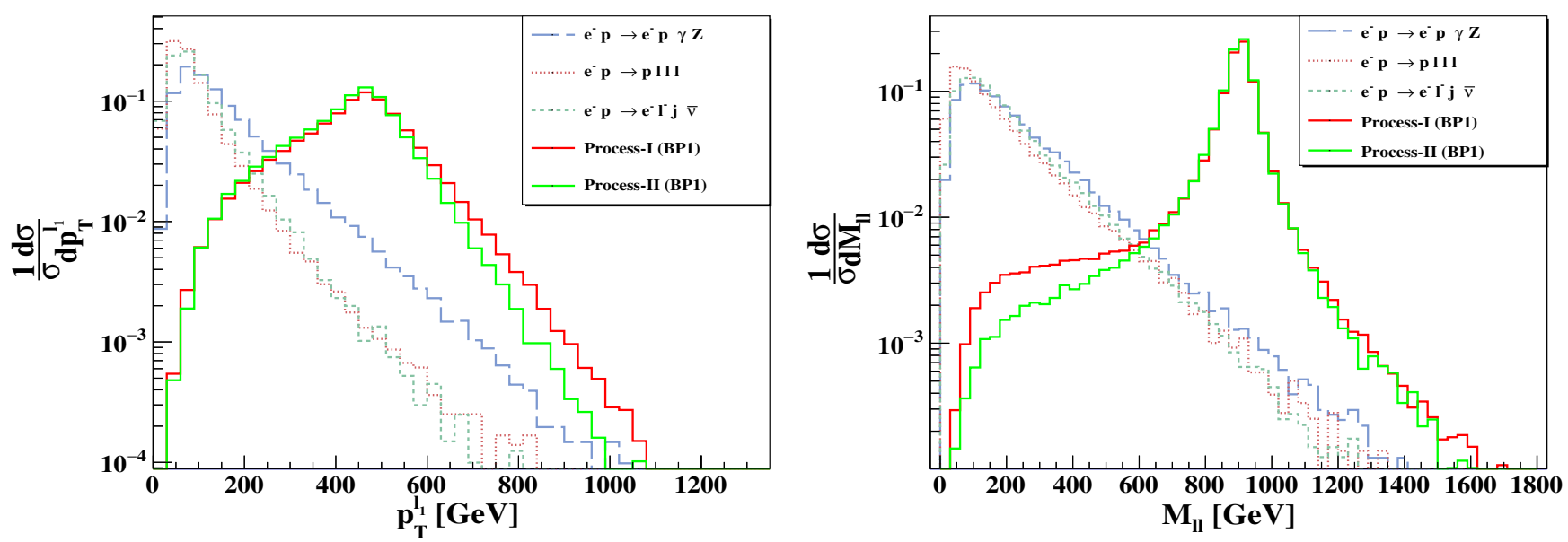

FIG. 4. The normalized distribution of $p_{T}$ of leading charged lepton (left) and the invariant mass $\left(M_{l l}\right)$ of the same-sign lepton pair (right) for both the signal and SM background.

A3. We also demand that the invariant mass $M_{l l}$ of samesign lepton pair is large, with $M_{l l}>800 \mathrm{GeV}$. This cut reduces the background significantly.

In fact, the only relevant SM background events that remain after cut $\mathbf{A} \mathbf{3}$ are from the process $e^{-} p \rightarrow e^{-} l^{+} l^{-} j$. The suppression in the signal cross section for all the three benchmark points is about $50 \%$ for the event selection cuts, as can be seen in Table IV.

\section{B. Signal II $(\geq \mathbf{2 l}+\geq \mathbf{1} j)$}

We now consider the multilepton channel where we have at least two charged leptons in the final state and both need to be of the same sign and flavor. This would correspond to the inclusive search of the signal for a singly-produced doubly-charged Higgs boson coming from both process I and process II. The combination of both the processes for the inclusive search would mean that one avoids kinematic selection of events that could potentially reduce contribution from any of the particular processes. This, on the other hand, would mean that there would be additional sources of background for the final state under consideration from the ones discussed in the previous subsection which are listed below.

(1) The irreducible background coming from $e^{-} p \rightarrow$ $e^{-} l^{-} j \overline{\nu_{l}}$. Again, for the same-sign, same-flavor requirement of the charged-lepton pair, the signal topology ensures that $l=e$.

(2) To add to the fake-rate contribution, we must consider the process $e^{-} p \rightarrow e^{-} j \gamma$, where $\gamma$ can fake the charged lepton $e^{-}$.

(3) In addition to fake rates, we note that charge mismeasurement can also lead to potential backgrounds which mimic the signal. We evaluate $e^{-} p \rightarrow e^{-} e^{+} j \nu$ process, where we consider a conservative $0.1 \%$ [127] as the charge mismeasurement or charge-flip rate/probability in the detector.
(4) We also estimate the background that arises due to the jet faking a lepton which as before is assumed to be $0.1 \%$.

As before, B0 and B1 discussed below define the selection of events for the signal in consideration.

B0. As in signal I, the final state leptons and jets must satisfy a minimum requirement for the transverse momenta and their pseudorapidity, given by $p_{T}^{j}>40 \mathrm{GeV}, p_{T}^{l}>10 \mathrm{GeV},\left|\eta^{j, l}\right| \leq 4.5$.

B1. We then demand that we select only those events which have at least two charged leptons and a minimum of one jet, which defines the signal as $\geq 2 l+\geq 1 j$.

Similar to the previous analysis, we again implement a few similar cuts on the events to suppress the background and improve signal sensitivity.

B2. The events satisfying $\mathbf{B 0}$ and $\mathbf{B} \mathbf{1}$ are then required to contain two leptons of same sign and same flavor. The kinematic properties of these charged leptons is expected to be similar to what was observed in Fig. 4, and therefore, we impose a cut on the $p_{T}$ of the leading and subleading charged lepton as $p_{T}^{l_{i}}>50 \mathrm{GeV}(i=1,2)$ while the leading jet must satisfy $p_{T}^{j_{1}}>50 \mathrm{GeV}$.

B3. To utilize the hardness of the invariant mass distribution of the same sign-same-flavor dilepton for the signal in suppressing the background, we further demand that $M_{l l}>800 \mathrm{GeV}$.

In Tables V and VI, we show the cut flow of the SM background and signal for the aforementioned cuts. As before, the most selective of all the cuts which improves the signal over the SM background is B3. However, although the $(\geq 2 l+\geq 1 j)$ inclusive channel gets contributions from both the processes shown in Fig. 1, the signal-to-background ratio is still inferior to the trilepton channel. The reason for this is because of the size of the continuum background $e^{-} l^{-} j \bar{\nu}$. 
TABLE V. Cut-flow table of the cross section for the relevant SM background channels for the cuts $\mathbf{B 0}-\mathbf{B 3}$ as mentioned in the text at the FCC-eh collider with $3.46 \mathrm{TeV}$ center-of-mass energy. We assume $l=e$ or $\mu$.

\begin{tabular}{lccccc}
\hline \hline $\begin{array}{l}\text { SM backgrounds } \\
\text { for FCC-eh }\end{array}$ & \multicolumn{5}{c}{$\begin{array}{c}\text { Effective cross section after } \\
\text { applying cuts }(\mathrm{fb})\end{array}$} \\
\cline { 1 - 5 } Channels & & B0 & B1 & B2 & B3 \\
\cline { 1 - 5 } \cline { 3 - 6 }$e^{-} p \rightarrow e^{-} l^{-} j \bar{\nu}$ & & 659.2 & 447.5 & 56.7 & 1.24 \\
$e^{-} p \rightarrow e^{-} j \gamma$ & $2.24 \times 10^{3}$ & 0.06 & $\ldots$ & $\ldots$ \\
$e^{-} p \rightarrow j l l l$ & & 53.13 & 41.2 & 40.4 & 0.1 \\
$e^{-} p \rightarrow e^{+} e^{-} j \nu$ & 346.0 & 0.22 & 0.04 & $6.8 \times 10^{-4}$ \\
$e^{-} p \rightarrow e^{-} j j$ & $104.08 \times 10^{3}$ & 327.18 & 37.0 & 0.37 \\
Total background & & & & 1.71 \\
\hline \hline
\end{tabular}

TABLE VI. Cut-flow table of cross section for signal for the cuts $\mathbf{B 0}-\mathbf{B 3}$ as mentioned in the text at the FCC-eh collider with $3.46 \mathrm{TeV}$ center-of-mass energy. Here, we consider $\mathrm{BR}\left(H^{ \pm \pm} \rightarrow e^{ \pm} e^{ \pm}\right)=100 \%$.

\begin{tabular}{|c|c|c|c|c|c|c|}
\hline \multicolumn{3}{|c|}{ Signal for FCC-eh } & \multicolumn{4}{|c|}{$\begin{array}{l}\text { Effective cross section after } \\
\text { applying cuts (fb) }\end{array}$} \\
\hline Process & Signal & BP & B0 & B1 & B2 & B3 \\
\hline $\mathrm{I}+\mathrm{II}$ & $\geq 2 l+\geq 1 j$ & BP1 & 0.36 & 0.29 & 0.24 & 0.21 \\
\hline $\mathrm{I}+\mathrm{II}$ & $\geq 2 l+\geq 1 j$ & BP2 & 0.22 & 0.18 & 0.14 & 0.13 \\
\hline $\mathrm{I}+\mathrm{II}$ & $\geq 2 l+\geq 1 j$ & BP3 & 0.13 & 0.11 & 0.09 & 0.08 \\
\hline
\end{tabular}

\section{C. $\mu \mu$ channel}

An important point which we should highlight here is the fact that so far we have only considered the electron flavor for the same-sign leptons in the decay of the doublycharged Higgs boson, while in principle, it could decay to any of the lepton flavors depending on the Yukawa structure $Y_{i j}^{\Delta}$. However, at an $e^{-} p$ machine, the SM background with two same-sign negatively-charged leptons would be dominated by the electron pair. Thus, for a suitable choice of $Y_{e e}^{\Delta}$ and $Y_{\mu \mu}^{\Delta}$, we could essentially have the signal containing two same-sign muons in the final state which would be relatively background free after the cuts we put. To put this fact in perspective, we list in Table VII the signal events for both the final states in consideration, written explicitly as $e^{+}+2 \mu^{-}+\geq 1 j$ and

TABLE VII. Signal cross section after all the cuts for $e^{+}+2 \mu^{-}+\geq 1 j(\mathbf{A 0}-\mathbf{A 3})$ and $2 \mu^{-}+\geq 1 j+\geq 0 l$ (B0-B3) mentioned in the text. Here, we consider $\operatorname{BR}\left(H^{ \pm \pm} \rightarrow e^{ \pm} e^{ \pm}\right)=$ $\operatorname{BR}\left(H^{ \pm \pm} \rightarrow \mu^{ \pm} \mu^{ \pm}\right)=50 \%$.

\begin{tabular}{llcl}
\hline \hline & \multicolumn{3}{c}{$\sigma(\mathrm{fb})$} \\
\cline { 2 - 4 } Signal & BP1 & BP2 & BP3 \\
\hline$e^{+}+2 \mu^{-}+\geq 1 j$ & 0.06 & 0.04 & 0.02 \\
$2 \mu^{-}+\geq 1 j+\geq 0 l$ & 0.12 & 0.08 & 0.05 \\
\hline \hline
\end{tabular}

$2 \mu^{-}+\geq 1 j+\geq 0 l$, after all the cuts mentioned before. Here, we assume that both the Yukawa couplings $Y_{e e}^{\Delta}$ and $Y_{\mu \mu}^{\Delta}$ are nonzero and of equal strength with the values given by Table II for each benchmark point, while satisfying the constraint given in Eq. (24), such that the $\mathrm{H}^{--}$ decay is now equally shared between the electron and muon modes, which means a $50 \%$ branching ratio for the $\mu \mu$ channel. The presence of a large $Y_{e e}^{\Delta}$, on the other hand, ensures a significant production cross section section of the doubly-charged Higgs boson.

Note that the leading contribution for the SM background in the $\mu \mu$ channel would arise from the subprocesses: $e^{-} p \rightarrow \nu_{l} Z W^{-} j$ and $e^{-} p \rightarrow e^{-} Z W^{-} j$, with both the $W$ and $Z$ bosons decaying into the muon channels. The parton-level cross section for these processes is itself at the level of $\sim 10^{-2} \mathrm{fb}$, and assuming that similar cut efficiencies for the background would apply as in the case of the ee mode, the background numbers would get further suppressed by a factor of $\sim \mathcal{O}\left(10^{-3}\right)$, rendering the background almost negligible for the $\mu \mu$ channel.

Therefore, the cross sections for the signal events given in Table VII imply a nearly background-free signal of $\sim 5-10$ events with an integrated luminosity of about $100 \mathrm{fb}^{-1}$. Thus, our predictions are more conservative when including only electron flavor in our analysis, which is still significantly robust in the trilepton channel for mass values of the doubly-charged scalar we have chosen. We show this by presenting the signal significance in the following subsection.

\section{Signal significance}

In determining the statistical significance of the signal we have used the following general expression [128]:

$$
\mathcal{S}=\sqrt{2\left[(s+b) \ln \left(1+\frac{s}{b}\right)-s\right]},
$$

where $s$ and $b$ denote the number of signal and background events (i.e., cross section times integrated luminosity), respectively, after all selection cuts are applied. Note that the formula (25) reduces to the more well-known form $\mathcal{S}=s / \sqrt{b}$ in the limit $s \ll b$. In Table VIII, we show the required integrated luminosity for either $3 l$ or $\geq 2 l$ channel to be observed with $3 \sigma$ and $5 \sigma$ statistical significance, where we consider $l=e$ only. Owing to the better $s / b$ ratio for the trilepton channel, one is clearly sensitive to the new physics signal in the trilepton channel with a relatively smaller data set when compared to the inclusive channel. However, both the channels provide a robust discovery prospect of the doubly-charged Higgs boson at FCC-eh, provided we have a sizable Yukawa coupling to the electron flavor, $Y_{e e}^{\Delta} \sim \mathcal{O}(1)$. Now, if the signal is driven by the $\mu \mu$ channel too, with the decay probability of the doublycharged Higgs boson being $50 \%$ in the muon mode, then 
TABLE VIII. Required integrated luminosity for achieving a $3 \sigma$ and $5 \sigma$ excess of the $3 l(\geq 2 l)$ signal, where the signal contains at least two electrons $\left(e^{-} e^{-}\right)$. Here, we consider $\mathrm{BR}\left(H^{ \pm \pm} \rightarrow e^{ \pm} e^{ \pm}\right)=100 \%$.

\begin{tabular}{llc}
\hline \hline & \multicolumn{2}{c}{$\int \mathcal{L} d t\left(\mathrm{fb}^{-1}\right)$} \\
\cline { 2 - 3 } Benchmark points & $\mathcal{S}=3 \sigma$ & $\mathcal{S}=5 \sigma$ \\
\hline BP1 & $118(354)$ & $328(982)$ \\
BP2 & $245(941)$ & $681(2615)$ \\
BP3 & $520(2034)$ & $1444(5650)$ \\
\hline \hline
\end{tabular}

the required integrated luminosity for discovery reduces drastically. In fact, assuming a conservative background of around $10^{-5} \mathrm{fb}$ in the $\mu \mu$ channel as discussed above and using the trilepton signal data from Table VII, we find that for BP1 discovery at $5 \sigma$ statistical significance, the required integrated luminosity is just $27 \mathrm{fb}^{-1}$, while it is $43 \mathrm{fb}^{-1}$ and $95 \mathrm{fb}^{-1}$ for BP2 and BP3, respectively.

To highlight the sensitivity of the FCC-eh to a doublycharged Higgs mass at a given integrated luminosity, we should know what values of the Yukawa coupling $Y_{e e}^{\Delta}$ yield the required $H^{--}$production cross section. This is shown in Fig. 5 where we plot the $2 \sigma$ and $3 \sigma$ reach for the doublycharged Higgs boson in the trilepton (signal I) and the inclusive dilepton (signal II) channels, and show the range of the Yukawa coupling values which would achieve the signal significance for a fixed integrated luminosity of $3 \mathrm{ab}^{-1}$. This is for the scenario where we demand that $H^{--}$ decays to $e^{-} e^{-}$with $100 \%$ branching ratio. The sensitivity curves are obtained using Eq. (25) with the signal and background values obtained from the cut-based analysis described above. Figure 5 in turn also gives us information on the maximum allowed value of the triplet VEV (corresponding to the minimum value of $Y_{e e}^{\Delta}$ accessible), so as to observe a signal for the doubly-charged Higgs boson at the FCC-eh. The vertical line in Fig. 5 shows the current lower limit on the doubly-charged scalar mass from the $\sqrt{s}=13 \mathrm{TeV}$ LHC data, assuming $100 \%$ branching ratio of the $H^{--}$decay to same-sign electron pair $[97,98]$. Strictly speaking, this bound is applicable only when the $H^{ \pm \pm} \rightarrow e^{ \pm} e^{ \pm}$decay is prompt, which requires $Y_{e e}^{\Delta} \gtrsim 10^{-7}$ (but practically indistinguishable from zero in the linear $Y_{e e}^{\Delta}$-scale of Fig. 5). The green-shaded region is excluded by LEP data on the Bhabha scattering process $e^{+} e^{-} \rightarrow$ $e^{+} e^{-}[113,115]$ as given by Eq. (23). The direct search limits from HERA [50], LEP [112-114], and Tevatron $[129,130]$ are only relevant for smaller $M_{H^{ \pm \pm}}$well below the mass range shown here and are anyway superseded by the LHC limits. It is worth noting that even the highluminosity phase of the $\sqrt{s}=14 \mathrm{TeV}$ LHC cannot improve the mass reach beyond $1 \mathrm{TeV}$ [69], and therefore, the FCC-eh provides a unique opportunity to directly probe the Yukawa coupling of heavier doubly-charged scalars to electrons. Only the proton-proton mode of FCC (FCC-hh) will be able to achieve a higher sensitivity in $M_{H^{ \pm \pm}}$up to about $6 \mathrm{TeV}$ [76], independent of $Y_{e e}^{\Delta}$, as long as $H^{ \pm \pm}$ dominantly decays to same-sign lepton pairs. On the other hand, the future $e^{+} e^{-}$colliders like ILC (or CLIC) might be able to cover a similar (or larger) parameter space in the direct (or indirect) search channel, as compared to the FCCeh reach shown in Fig. 5, depending on the center-of-mass energy [93].

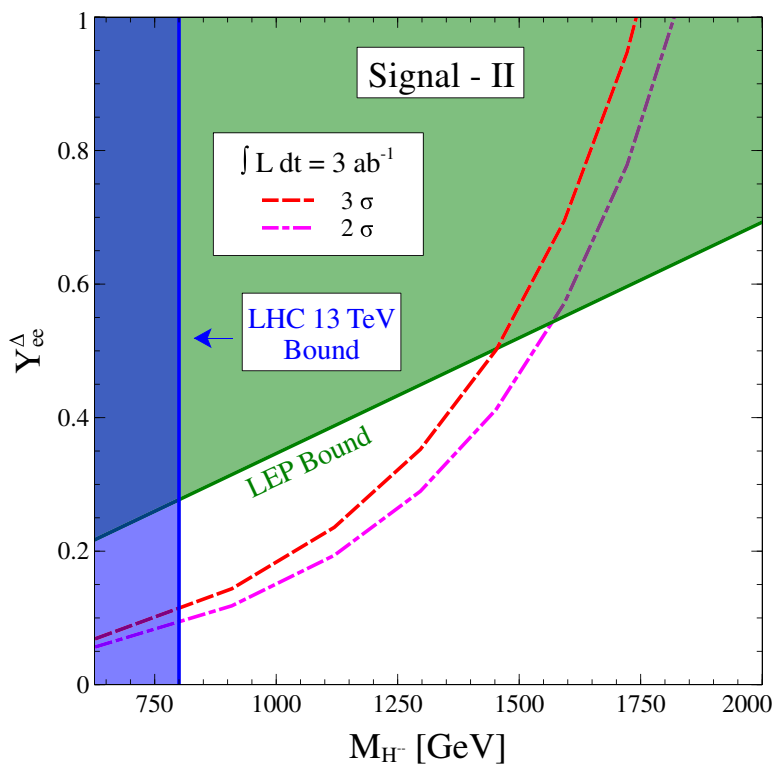

FIG. 5. The $2 \sigma$ and $3 \sigma$ sensitivity reach of $Y_{e e}^{\Delta}$ at FCC-eh as a function of the doubly-charged Higgs mass $M_{H^{--}}$. The left panel is for signal I and the right panel is for signal II discussed in the text. The vertical (blue) shaded region is excluded from the current lower bound on $M_{H^{ \pm \pm}}$from the $\sqrt{s}=13 \mathrm{TeV}$ LHC data. The green-shaded region is excluded by the LEP constraints on the Bhabha scattering process. Here, we have considered $\operatorname{BR}\left(H^{ \pm \pm} \rightarrow e^{ \pm} e^{ \pm}\right)=100 \%$. 


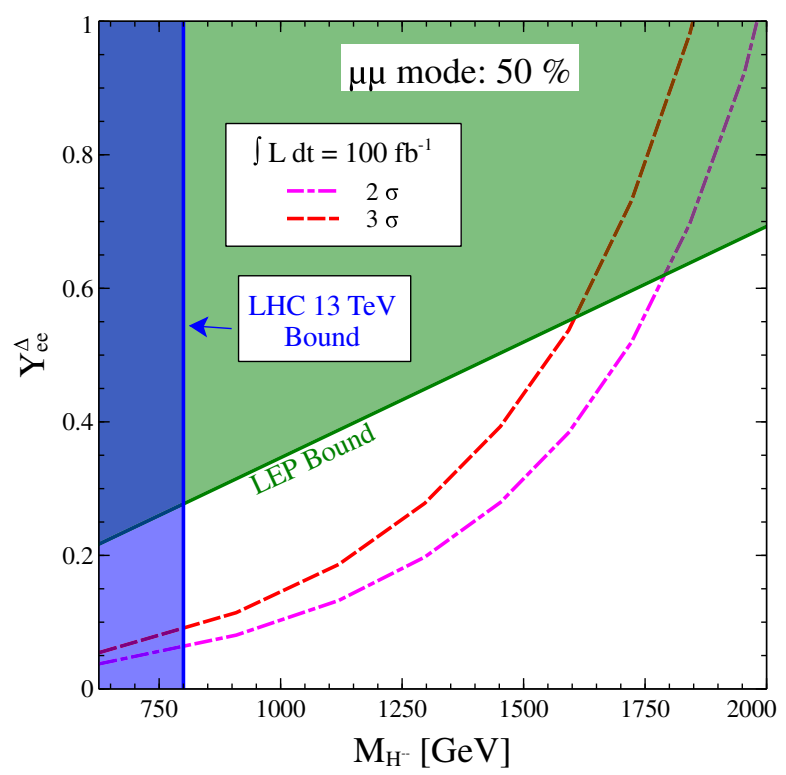

FIG. 6. The sensitivity reach of $Y_{e e}^{\Delta}$ at FCC-eh as a function of the doubly-charged Higgs mass $M_{H^{ \pm \pm}}$for signal I with $\operatorname{BR}\left(H^{--} \rightarrow \mu^{-} \mu^{-}\right)=50 \%$. The vertical (blue) shaded region is excluded from the current lower bound on $M_{H^{ \pm \pm}}$from the $\sqrt{s}=$ $13 \mathrm{TeV}$ LHC data. The green-shaded region is excluded by the LEP constraints on the Bhabha scattering process.

The more robust and striking channel of discovery would however be the $\mu^{-} \mu^{-}$channel, as illustrated in Fig. 6, which is relatively background free, barring very low rates from associated $W^{-} Z$ productions. As a large production rate for the doubly-charged Higgs boson is a necessity, we ensure a $50 \%$ decay probability of the $H^{ \pm \pm}$in the $\mu \mu$ mode while respecting the bound of Eq. (24). We show the much improved sensitivity reach using the signal rates for $e^{+}+2 \mu^{-}+\geq 1 j$ final state, where a similar $2 \sigma$ or $3 \sigma$ range as in Fig. 5 can now be attained with a much smaller integrated luminosity of $100 \mathrm{fb}^{-1}$. A similar sensitivity is also expected in the inclusive $2 \mu^{-}+\geq 1 j+\geq 0$ l channel. This reach can be further improved if the signal significance of both the $e e$ and $\mu \mu$ channels (observed independently) are combined together.

\section{CONCLUSION}

We have analyzed the discovery prospect of a doublycharged Higgs boson at the newly proposed FCC-eh electron-proton collider, operating with beam energies $E_{e}=60 \mathrm{GeV}$ for electron beam and $E_{p}=50 \mathrm{TeV}$ for proton beam. As the LHC would have limited sensitivity to a doubly-charged Higgs mass, even with the high-luminosity phase, searching for heavier doubly-charged scalars would be a challenge. A type-II seesaw-based scenario for neutrino mass generation with a very tiny triplet VEV would imply a substantially large Yukawa coupling of the scalar to the charged leptons. Neither the LHC nor an $e^{+} e^{-}$ machine could gain from this, unless the collisions are of the $e^{-} e^{-}$type [86], where the production of doubly charged Higgs boson has a direct dependency on Yukawa. However, the proposed FCC machine, where the collisions are between an electron and a proton beam (FCC-eh) would open up the possibility of looking for these heavier doublycharged states through their single on shell production in association with lepton and a jet.

We have studied two types of final states, namely, $3 l+\geq 1 j$ and inclusive $\geq 2 l+\geq 1 j$, to probe $\mathrm{TeV}$-scale doublycharged scalars at FCC-eh. We consider a few representative benchmark points for a hybrid type-I + type-II seesaw with a small triplet VEV $v_{\Delta} \sim \mathcal{O}(0.1) \mathrm{eV}$ and large diagonal Yukawa couplings $Y_{e e}^{\Delta} \sim \mathcal{O}(1)$. The model is consistent with neutrino oscillation data, as well as, in agreement with the bounds from lepton flavor violating processes, such as, $\mu \rightarrow e \gamma$ and $\mu \rightarrow e e e$. We find that a doubly-charged Higgs boson with a mass around a TeV can be probed at a $3 \sigma$ significance in FCC-eh with $\mathcal{L} \sim$ $245 \mathrm{fb}^{-1}$ in the $3 l+\geq 1 j(l=e)$ mode. Moreover, higher mass values, up to about $2 \mathrm{TeV}$, could also be probed within a few years' of running of the FCC-eh. A more robust and striking channel would be observed with only $\mathcal{L}=$ $100 \mathrm{fb}^{-1}$ of data if the doubly-charged Higgs boson has a decay probability in the $\mu \mu$ mode, which is comparable, if not more than the ee mode. This reach can be further improved if the signal significance of both the $e e$ and $\mu \mu$ channels (observed independently) are combined.

\section{ACKNOWLEDGMENTS}

B.D., M. M., and S.K.R. thank the organizers of WHEPP-XIV at IIT, Kanpur where this work was initiated, and the organizers of SUSY 2017 at TIFR, Mumbai where part of this work was done, for the local hospitality. S. K. would like to thank Subhadeep Mondal for help with numerical aspects at the initial stage of the work. The work of B. D. is supported by the US Department of Energy under Grant No. DE-SC0017987. M. M. acknowledges the support of DST-INSPIRE research Grant No. IFA14-PH99. The work of S. K. R. was partially supported by funding available from the Department of Atomic Energy, Government of India, for the Regional Centre for Accelerator-based Particle Physics (RECAPP), HarishChandra Research Institute. S. K. would like to acknowledge support from the Department of Atomic Energy (DAE) Neutrino Project under the plan project of Harish-Chandra Research Institute and the European Union's Horizon 2020 research and innovation programme Invisibles Plus RISE under the Marie Sklodowska-Curie Grant No. 690575. 
[1] P. F. De Salas, S. Gariazzo, O. Mena, C. A. Ternes, and M. Tortola, Front. Astron. Space Sci. 5, 36 (2018).

[2] R. N. Mohapatra and A. Y. Smirnov, Annu. Rev. Nucl. Part. Sci. 56, 569 (2006).

[3] S. Weinberg, Phys. Rev. Lett. 43, 1566 (1979).

[4] F. Wilczek and A. Zee, Phys. Rev. Lett. 43, 1571 (1979).

[5] E. Ma, Phys. Rev. Lett. 81, 1171 (1998).

[6] P. Minkowski, Phys. Lett. 67B, 421 (1977).

[7] R. N. Mohapatra and G. Senjanović, Phys. Rev. Lett. 44, 912 (1980).

[8] T. Yanagida, Conf. Proc. C 7902131, 95 (1979).

[9] M. Gell-Mann, P. Ramond, and R. Slansky, Conf. Proc. C 790927, 315 (1979).

[10] S. L. Glashow, NATO Sci. Ser. B 61, 687 (1980).

[11] W. Konetschny and W. Kummer, Phys. Lett. 70B, 433 (1977).

[12] J. Schechter and J. W. F. Valle, Phys. Rev. D 22, 2227 (1980).

[13] M. Magg and C. Wetterich, Phys. Lett. 94B, 61 (1980).

[14] T. P. Cheng and L.-F. Li, Phys. Rev. D 22, 2860 (1980).

[15] G. Lazarides, Q. Shafi, and C. Wetterich, Nucl. Phys. B181, 287 (1981).

[16] R. N. Mohapatra and G. Senjanović, Phys. Rev. D 23, 165 (1981).

[17] R. Foot, H. Lew, X. G. He, and G. C. Joshi, Z. Phys. C 44, 441 (1989).

[18] R. N. Mohapatra and J. C. Pati, Phys. Rev. D 11, 566 (1975).

[19] R. N. Mohapatra and J. C. Pati, Phys. Rev. D 11, 2558 (1975).

[20] G. Senjanović and R. N. Mohapatra, Phys. Rev. D 12, 1502 (1975).

[21] R. N. Mohapatra, Phys. Rev. Lett. 56, 561 (1986).

[22] S. Nandi and U. Sarkar, Phys. Rev. Lett. 56, 564 (1986).

[23] R. N. Mohapatra and J. W. F. Valle, Phys. Rev. D 34, 1642 (1986).

[24] M. Malinsky, J. C. Romao, and J. W. F. Valle, Phys. Rev. Lett. 95, 161801 (2005).

[25] S. K. Kang and C. S. Kim, Phys. Lett. B 646, 248 (2007).

[26] M. B. Gavela, T. Hambye, D. Hernandez, and P. Hernandez, J. High Energy Phys. 09 (2009) 038.

[27] P. S. B. Dev and A. Pilaftsis, Phys. Rev. D 86, 113001 (2012).

[28] S.S. C. Law and K. L. McDonald, Phys. Rev. D 87, 113003 (2013).

[29] Y. Cai, J. Herrero-Garcia, M. A. Schmidt, A. Vicente, and R. R. Volkas, Front. Phys. 5, 63 (2017).

[30] A. Atre, T. Han, S. Pascoli, and B. Zhang, J. High Energy Phys. 05 (2009) 030.

[31] F. F. Deppisch, P. S. B. Dev, and A. Pilaftsis, New J. Phys. 17, 075019 (2015).

[32] T. Golling et al., CERN Yellow Rep. 3, 441 (2017).

[33] Y. Cai, T. Han, T. Li, and R. Ruiz, Front. Phys. 6, 40 (2018).

[34] J. de Blas et al., arXiv:1812.02093.

[35] J. L. A. Fernandez et al. (LHeC Study Group), J. Phys. G 39, 075001 (2012).

[36] Y. C. Acar, A. N. Akay, S. Beser, A. C. Canbay, H. Karadeniz, U. Kaya, B. B. Oner, and S. Sultansoy, Nucl. Instrum. Methods Phys. Res., Sect. A 871, 47 (2017).
[37] G. Azuelos, M. D’Onofrio, O. Fischer, and J. Zurita, Proc. Sci., DIS2018 (2018) 190.

[38] L. Han, X.-G. He, M. Wen-Gan, W. Shao-Ming, and Z. Ren-You, J. High Energy Phys. 09 (2010) 023.

[39] C. Blaksley, M. Blennow, F. Bonnet, P. Coloma, and E. Fernandez-Martinez, Nucl. Phys. B852, 353 (2011).

[40] L. Duarte, G. A. González-Sprinberg, and O. A. Sampayo, Phys. Rev. D 91, 053007 (2015).

[41] S. Mondal and S. K. Rai, Phys. Rev. D 93, 011702 (2016).

[42] M. Lindner, F. S. Queiroz, W. Rodejohann, and C. E. Yaguna, J. High Energy Phys. 06 (2016) 140.

[43] S. Mondal and S. K. Rai, Phys. Rev. D 94, 033008 (2016).

[44] S. Antusch, E. Cazzato, and O. Fischer, Int. J. Mod. Phys. A 32, 1750078 (2017).

[45] D. Curtin, K. Deshpande, O. Fischer, and J. Zurita, J. High Energy Phys. 07 (2018) 024.

[46] S. Mandal, M. Mitra, and N. Sinha, Phys. Rev. D 98, 095004 (2018).

[47] A. Das, S. Jana, S. Mandal, and S. Nandi, Phys. Rev. D 99, 055030 (2019).

[48] S.-Y. Li, Z.-G. Si, and X.-H. Yang, arXiv:1811.10313.

[49] E. Accomando and S. Petrarca, Phys. Lett. B 323, 212 (1994).

[50] A. Aktas et al. (H1 Collaboration), Phys. Lett. B 638, 432 (2006).

[51] C.-X. Yue, S. Zhao, and W. Ma, Nucl. Phys. B784, 36 (2007).

[52] P. A. R. Ade et al. (Planck Collaboration), Astron. Astrophys. 594, A13 (2016).

[53] J. A. Grifols, A. Mendez, and G. A. Schuler, Mod. Phys. Lett. A 04, 1485 (1989).

[54] J. F. Gunion, J. Grifols, A. Mendez, B. Kayser, and F. I. Olness, Phys. Rev. D 40, 1546 (1989).

[55] M. Muhlleitner and M. Spira, Phys. Rev. D 68, 117701 (2003).

[56] A. G. Akeroyd and M. Aoki, Phys. Rev. D 72, 035011 (2005).

[57] T. Han, B. Mukhopadhyaya, Z. Si, and K. Wang, Phys. Rev. D 76, 075013 (2007).

[58] W. Chao, Z.-G. Si, Z.-z. Xing, and S. Zhou, Phys. Lett. B 666, 451 (2008).

[59] P. F. Perez, T. Han, G.-y. Huang, T. Li, and K. Wang, Phys. Rev. D 78, 015018 (2008).

[60] F. del Aguila and J. A. Aguilar-Saavedra, Nucl. Phys. B813, 22 (2009).

[61] A. G. Akeroyd and C.-W. Chiang, Phys. Rev. D 80, 113010 (2009).

[62] A. G. Akeroyd, C.-W. Chiang, and N. Gaur, J. High Energy Phys. 11 (2010) 005.

[63] A. Melfo, M. Nemevsek, F. Nesti, G. Senjanović, and Y. Zhang, Phys. Rev. D 85, 055018 (2012).

[64] A. Alloul, M. Frank, B. Fuks, and M. R. de Traubenberg, Phys. Rev. D 88, 075004 (2013).

[65] E. J. Chun and P. Sharma, Phys. Lett. B 728, 256 (2014).

[66] F. del Águila and M. Chala, J. High Energy Phys. 03 (2014) 027.

[67] G. Bambhaniya, J. Chakrabortty, J. Gluza, M. Kordiaczyńska, and R. Szafron, J. High Energy Phys. 05 (2014) 033. 
[68] P. S. B. Dev, R. N. Mohapatra, and Y. Zhang, J. High Energy Phys. 05 (2016) 174.

[69] M. Mitra, S. Niyogi, and M. Spannowsky, Phys. Rev. D 95, 035042 (2017).

[70] D. K. Ghosh, N. Ghosh, I. Saha, and A. Shaw, Phys. Rev. D 97, 115022 (2018).

[71] T. Li, J. High Energy Phys. 09 (2018) 079.

[72] D. Borah, B. Fuks, D. Goswami, and P. Poulose, Phys. Rev. D 98, 035008 (2018).

[73] S. Antusch, E. Cazzato, O. Fischer, A. Hammad, and K. Wang, J. High Energy Phys. 10 (2018) 067.

[74] A. Crivellin, M. Ghezzi, L. Panizzi, G. M. Pruna, and A. Signer, Phys. Rev. D 99, 035004 (2019).

[75] P. S. B. Dev and Y. Zhang, J. High Energy Phys. 10 (2018) 199.

[76] Y. Du, A. Dunbrack, M. J. Ramsey-Musolf, and J.-H. Yu, J. High Energy Phys. 01 (2019) 101.

[77] K. S. Babu and S. Jana, Phys. Rev. D 95, 055020 (2017).

[78] K. Huitu, J. Maalampi, A. Pietila, and M. Raidal, Nucl. Phys. B487, 27 (1997).

[79] J. Maalampi and N. Romanenko, Phys. Lett. B 532, 202 (2002).

[80] S. Kanemura, K. Yagyu, and H. Yokoya, Phys. Lett. B 726, 316 (2013).

[81] C. Englert, E. Re, and M. Spannowsky, Phys. Rev. D 88, 035024 (2013).

[82] B. Dutta, R. Eusebi, Y. Gao, T. Ghosh, and T. Kamon, Phys. Rev. D 90, 055015 (2014).

[83] S. Kanemura, M. Kikuchi, K. Yagyu, and H. Yokoya, Phys. Rev. D 90, 115018 (2014).

[84] G. Bambhaniya, J. Chakrabortty, J. Gluza, T. Jelinski, and R. Szafron, Phys. Rev. D 92, 015016 (2015).

[85] A. M. Sirunyan et al. (CMS Collaboration), Phys. Rev. Lett. 120, 081801 (2018).

[86] B. Mukhopadhyaya and S. K. Rai, Phys. Lett. B 633, 519 (2006).

[87] C.-S. Chen, C.-Q. Geng, and D. V. Zhuridov, Eur. Phys. J. C 60, 119 (2009).

[88] W. Rodejohann, Phys. Rev. D 81, 114001 (2010).

[89] C.-X. Yue, X.-S. Su, J. Zhang, and J. Wang, Commun. Theor. Phys. 56, 709 (2011).

[90] W. Rodejohann and H. Zhang, Phys. Rev. D 83, 073005 (2011).

[91] T. Nomura, H. Okada, and H. Yokoya, Nucl. Phys. B929, 193 (2018).

[92] P. Agrawal, M. Mitra, S. Niyogi, S. Shil, and M. Spannowsky, Phys. Rev. D 98, 015024 (2018).

[93] P. S. B. Dev, R. N. Mohapatra, and Y. Zhang, Phys. Rev. D 98, 075028 (2018).

[94] P. S. B. Dev, M. J. Ramsey-Musolf, and Y. Zhang, Phys. Rev. D 98, 055013 (2018).

[95] A. G. Akeroyd, M. Aoki, and H. Sugiyama, Phys. Rev. D 79, 113010 (2009).

[96] P. S. B. Dev, C. M. Vila, and W. Rodejohann, Nucl. Phys. B921, 436 (2017).

[97] V. Khachatryan et al. (CMS Collaboration), A search for doubly-charged Higgs boson production in three and four lepton final states at $\sqrt{s}=13 \mathrm{TeV}$, CERN Technical Report No CMS-PAS-HIG-16-036, 2017.
[98] M. Aaboud et al. (ATLAS Collaboration), Eur. Phys. J. C 78, 199 (2018).

[99] J. Barry and W. Rodejohann, J. High Energy Phys. 09 (2013) 153.

[100] G. Bambhaniya, P. S. B. Dev, S. Goswami, and M. Mitra, J. High Energy Phys. 04 (2016) 046.

[101] A. Arhrib, R. Benbrik, M. Chabab, G. Moultaka, M. C. Peyranere, L. Rahili, and J. Ramadan, Phys. Rev. D 84, 095005 (2011).

[102] E. J. Chun, H. M. Lee, and P. Sharma, J. High Energy Phys. 11 (2012) 106.

[103] P. S. B. Dev, D. K. Ghosh, N. Okada, and I. Saha, J. High Energy Phys. 03 (2013) 150; 05 (2013) 049(E).

[104] A. Kobakhidze and A. Spencer-Smith, J. High Energy Phys. 08 (2013) 036.

[105] M. Chabab, M. C. Peyranère, and L. Rahili, Phys. Rev. D 93, 115021 (2016).

[106] N. Haba, H. Ishida, N. Okada, and Y. Yamaguchi, Eur. Phys. J. C 76, 333 (2016).

[107] D. Das and A. Santamaria, Phys. Rev. D 94, 015015 (2016).

[108] X.-J. Xu, Phys. Rev. D 94, 115025 (2016).

[109] S. Antusch, O. Fischer, A. Hammad, and C. Scherb, J. High Energy Phys. 02 (2019) 157.

[110] A. M. Baldini et al. (MEG Collaboration), Eur. Phys. J. C 76, 434 (2016).

[111] U. Bellgardt et al. (SINDRUM Collaboration), Nucl. Phys. B299, 1 (1988).

[112] J. Abdallah et al. (DELPHI Collaboration), Phys. Lett. B 552, 127 (2003).

[113] G. Abbiendi et al. (OPAL Collaboration), Phys. Lett. B 577, 93 (2003).

[114] P. Achard et al. (L3 Collaboration), Phys. Lett. B 576, 18 (2003).

[115] J. Abdallah et al. (DELPHI Collaboration), Eur. Phys. J. C 45, 273 (2006).

[116] L. Willmann et al., Phys. Rev. Lett. 82, 49 (1999).

[117] Y. Amhis et al. (HFLAV Collaboration), Eur. Phys. J. C 77, 895 (2017).

[118] A. Alloul, N. D. Christensen, C. Degrande, C. Duhr, and B. Fuks, Comput. Phys. Commun. 185, 2250 (2014).

[119] J. Alwall, R. Frederix, S. Frixione, V. Hirschi, F. Maltoni, O. Mattelaer, H. S. Shao, T. Stelzer, P. Torrielli, and M. Zaro, J. High Energy Phys. 07 (2014) 079.

[120] J. Pumplin, D. R. Stump, J. Huston, H. L. Lai, P. M. Nadolsky, and W. K. Tung, J. High Energy Phys. 07 (2002) 012 .

[121] T. Sjostrand, S. Mrenna, and P. Z. Skands, J. High Energy Phys. 05 (2006) 026.

[122] J. de Favereau, C. Delaere, P. Demin, A. Giammanco, V. Lemaítre, A. Mertens, and M. Selvaggi (DELPHES 3 Collaboration), J. High Energy Phys. 02 (2014) 057.

[123] M. Cacciari, G. P. Salam, and G. Soyez, J. High Energy Phys. 04 (2008) 063.

[124] M. Cacciari, G. P. Salam, and G. Soyez, Eur. Phys. J. C 72, 1896 (2012).

[125] G. Aad et al. (ATLAS Collaboration), Phys. Rev. D 83, 112001 (2011). 
[126] G. Aad et al. (ATLAS Collaboration), Electron efficiency measurements with the ATLAS detector using the 2015 LHC proton-proton collision data, CERN Technicl Report No. ATLAS-CONF-2016-024, 2016.

[127] V. Khachatryan et al. (CMS Collaboration), J. High Energy Phys. 04 (2016) 169.
[128] G. Cowan, K. Cranmer, E. Gross, and O. Vitells, Eur. Phys. J. C 71, 1554 (2011); 73, 2501(E) (2013).

[129] D. Acosta et al. (CDF Collaboration), Phys. Rev. Lett. 93, 221802 (2004).

[130] V. M. Abazov et al. (D0 Collaboration), Phys. Rev. Lett. 101, 071803 (2008). 